\title{
Review on Classification and Clustering using Fuzzy Neural Networks
}

\author{
Suprit Kulkarni \\ S.Y., M. E. Computer Engineering \\ SKN College of Engineering \\ Vadgaon, Pune (M.S), India
}

\author{
Kishore Honwadkar \\ Professor, Dept. of Computer Engineering \\ SKN College of Engineering \\ Vadgaon, Pune (M.S), India
}

\begin{abstract}
In data mining two important tasks involved are classification and clustering. In general, in classification the classifier assigns a class label from a set of predefined classes to a new input object. Whereas, given a set of objects, clustering creates different groups of these objects using some similarity measure. In the context of machine learning, classification is supervised learning and clustering is unsupervised learning. There are different approaches used for classification and clustering. In recent past many fuzzy neural networks have been proposed which can be employed for classification and clustering. Unlike other techniques, the fuzzy neural networks are quickly trainable, suitable for online training, provides soft decision, and capable of constructing nonlinear decision boundaries. All these benefits make them suitable for difficult real world problems involving classification and clustering. This paper provides review on recent fuzzy neural learning algorithms and mainly focusing on pattern/object classification and clustering.
\end{abstract}

\section{General Terms}

Pattern Classification; Data Mining; Neural Networks; Fuzzy Logic

\section{Keywords}

Classification; Clustering; Fuzzy Neural Network

\section{INTRODUCTION}

In spite of significant progress in computer-based pattern classification, recognition and clustering, living things seem to be more competent in solving such many difficult tasks which still cannot be handled effortlessly by computers. One of the many talented approaches to such problems using computer is the use of artificial neural networks. They have been successfully used in many pattern classification, recognition and clustering problems.

There are two main training strategies for pattern classification and clustering approaches; supervised and unsupervised learning [1].

In supervised learning, frequently referred as a pattern classification problem in which class labels are available with input patterns during training and the trained classifier constructs decision boundaries between classes that minimize misclassification.

Nevertheless, the unsupervised learning is often referred as a clustering problem. The patterns during training are unlabeled and the task is dividing a set of unlabeled patterns into few clusters using some suitable similarity measure. There are different similarity measures which are used for creating clusters of unlabeled data set. However, the most popular is a distance measure. The patterns or data samples which are similar are assigned to the same cluster, while the samples which differ considerably are placed in different clusters. Thus the clustering always performs partitioning of patterns in disconnected or overlapped clusters.

One more vital characteristic of human reasoning is the effortlessness in handling uncertain or vague data that appears in the real life. The traditional statistical approaches to pattern classification have been found inadequate in such circumstances and this deficiency has prompted the search for alternative solutions which allows representation of ambiguous data and more flexible labeling in classification problems.

The fuzzy sets were suggested as a way to remedy this difficulty. The fuzzy neural network (FNN) synergetic combination of fuzzy sets and artificial neural networks in the pattern classification and clustering has been studied by many researchers. The plasticity of fuzzy sets and the computational efficiency of artificial neural networks have caused a great amount of interest in the combination of these two techniques for solving pattern classification and clustering problems successfully with confirmed evidence.

Originally, Simpson proposed the fuzzy min-max (FMM) clustering and classification neural networks [2], [3], in which the classes are represented as fuzzy set hyperboxes in the $n$ dimensional pattern space. Subsequently, several researchers have proposed variants of the FMM neural networks suggesting different modifications in their learning algorithms.

This paper attempts to provide a brief review on FNNs. The review is covered in three parts: FNNs for classification, FNNs for clustering and General FNNs for classification and clustering.

The remainder of this paper is organized as follows. Section 2 gives a short description of original FMM algorithm for classification and covers survey of FNNs for classification. The Section 3 begins with little description of original FMM algorithm for clustering and subsequently reviews FNNs for clustering. Section 4 presents review on general FNNs which can be used in hybrid classification and clustering problems. Finally, the conclusions are outlined in Section 5.

\section{FNNs FOR CLASSIFICATION}

The FMM classification neural network [2] uses hyperbox fuzzy sets. A fuzzy set hyperbox is defined by its min-max points and the hyperbox fuzzy set membership function. A hyperbox min-max points define a region in the $n$-dimensional pattern space, and all patterns contained within the hyperbox have full class membership.

The hyperbox fuzzy sets are aggregated to form a single fuzzy set class during classification. Learning in the FMM classification neural network consists of creating, expanding and contracting hyperboxes in the pattern space. The learning begins by inputting an input pattern and finding the nearest hyperbox to that pattern that can expand (if necessary) to 
include the pattern. If a hyperbox cannot be found that meets the expansion criteria, a new hyperbox is created and added to the system. This augmentation process permits existing classes to be refined over time, and it allows addition of new classes without the need of retraining. One of the unwanted effects of hyperbox expansion is overlapping hyperboxes belonging to different classes. This overlapping causes ambiguity because patterns falling in the overlapping area fully belong to two or more different classes. A contraction process is utilized to get rid of any undesired hyperbox overlaps. The overlap is removed employing the minimum disturbance principle and only for hyperboxes that represent different classes. The trained network consists of three layers; input layer, hyperbox nodes layer and class nodes layer. The input layer nodes do not do any processing. The hyperbox nodes use the fuzzy membership function to deliver its output. Finally the class nodes perform aggregation of outputs of hyperboxes of that particular class to provide soft decision.

Most of the FNNs offer numerous benefits such as: soft decision, on line training, quick learning, and nonlinear separability. These advantages of FNNs with their proven record has caused a great amount of interest and their use in pattern classification and recognition problems [4], [6], [7], [8], [11], [14], [15], [16], [17], [18], [20], [21], [22], [23], [24], [25], [26].

In [4], a latest learning algorithm for the Simpson's fuzzy minmax neural network is proposed that improves the network performance. The classification result does not depend on the presentation order of the patterns in the training set. The proposed approach is particularly useful in classification problems and compared with Simpson's min-max model, fuzzy ARTMAP proposed by Carpenter, Grossberg et al. in 1992 and the classical multilayer perceptron neural network with backpropagation. The experiments are carried out on three classification problems including the real data for industrial diagnosis. Further to note, in [21], FMM neural network is employed for signature recognition.

Fuzzy hypersphere neural network (FHSNN) in [6] utilizes fuzzy sets as pattern classes in which each fuzzy set is an union of fuzzy set hyperspheres. Its performance is compared with other two FNNs and found to be superior with respect to the training time, recall time per pattern and the generalization ability.

Modular fuzzy hypersphere neural network (MFHSNN) in [15] offers higher degree of parallelism. Unlike in FHSNN, each module in MFHSNN is exposed to the patterns of only one class and trained without overlap test and removal, resulting in reduction of training time. Hence, each module captures peculiarity of only one particular class. The decreased training time allows the use of algorithm for big real database where new patterns can be added on the fly. The MFHSNN is found superior than FHSNN in terms of generalization and computational complexity.

Another variant of the FHSNN [11], the modified FHSNN is an extension to the FHSNN which is used for speech recognition successfully.

Fuzzy hyperline segment neural network (FHLSNN) in [8] utilizes fuzzy sets as pattern classes in which each fuzzy set is an union of fuzzy set hyperline segments. Its performance is compared with other FNNs using handwritten character data set after feature extraction invariant to rotation. The performance is found to be superior with respect to the training time, recall time per pattern and the generalization ability.
The contributors of [14] have utilized the modified FHLSNN for recognition of handwritten characters. The modified FHLSNN is a variant with few minor modifications in the learning algorithm of FHLSNN.

A fuzzy min-max neural network classifier with compensatory neurons (FMCNs) in [16], [17], and [18] uses hyperbox fuzzy sets to represent the pattern classes. It employs new compensatory neuron architecture. The notion of compensatory neuron is inspired from the reflex system of human biological neural network which takes over the control in dangerous situations. Compensatory neurons (CNs) emulate this behavior by getting activated whenever a trial sample falls in the overlapped regions amongst different classes. These $\mathrm{CNs}$ handle the hyperbox overlap and containment more efficiently. The minimal disturbance principal used by Simpson in [2], [3] for contraction to solve the problem of hyperbox overlaps is eliminated. The FMCN supports on line adaptation in a single pass and yields reduced classification and gradation errors. Its performance is less dependent on the initialization of maximum hyperbox size coefficient. The performance is demonstrated using several examples and comparing it with original FMM neural network [2] and general fuzzy min max (GFMM) neural network [5].

In [20], authors propose modifications to FMM to improve its classification performance particularly when a small number of large hyperboxes are formed. An Euclidean distance measure is introduced for predicting the target class associated with the new input pattern, in addition to measuring the fuzzy membership to the hyperboxes formed. A rule extraction algorithm is also included. Fuzzy if-then rules are extracted after pruning the network. To evaluate the usefulness of modified FMM neural network, two standard pattern classification problems are experimented. The results are compared with different methods published in the literature. Moreover, a fault detection and classification problem with a set of real sensor measurements collected from a power generation plant is evaluated.

In [22], a two-stage pattern classification and rule extraction system is proposed. The first stage is a modified FMM neural network pattern classifier. The modified FMM classifier extracts fuzzy if then rules. Whereas genetic-algorithm (GA)based rule extractor is used in the second stage. A "don't care" approach is adopted by the GA rule extractor to reduce the number of features in the extracted rules. Five yardstick problems and a real medical diagnosis task are used to experimentally evaluate the efficacy of the proposed system. Moreover, the bootstrap hypothesis analysis is carried to enumerate the results of the medical diagnosis task statistically. The proposed system could extract a set of compact and yet easily comprehensible rules maintaining a high classification performance.

A FMM neural network based on data core (DCFMN) is proposed in [23] for pattern classification. To classify the neuron of DCFMN a new membership function is proposed. While defining this membership function the noise, the geometric center of the hyperbox, and the data core are considered. The contraction process described by Simpson is eliminated. A new approach by using overlapped neuron with new membership function based on the data core is proposed and added to the neural network to represent the overlapping area of hyperboxes belonging to different classes. The performance of DCFMN is checked by a few standard datasets and compared with some traditional FNNs, such as FMM neural network, the GFMM neural network, and the FMCNs. 
The pipeline classification is evaluated using DCFMN and other classifiers. Authors observed that DCFMN outperforms.

In [24], a new approach to identify and classify complete fault conditions of induction motors using a hybrid FMM neural network and classification and regression tree (CART) is proposed. The hybrid model is abbreviated as FMM-CART. It includes the advantages of FMM and CART both. The data classification and rule extraction problems undertaken exploit these benefits. Several real experiments are conducted. The database is formed using motor current signature analysis. It comprises of stator current signatures under different motor conditions. Further, the signal harmonics from the power spectral density are extracted also used as discriminative input features for fault detection and classification. A wide-ranging list of induction motor fault conditions has been successfully classified with good classification accuracy. Moreover, useful explanatory rules in the form of a decision tree are also extracted. These rules help to analyze and understand different fault conditions of induction motors.

An enhanced fuzzy min-max (EFMM) neural network is proposed for pattern classification in [25]. The purpose is to beat a number of limitations of the original FMM neural network to get better classification performance. Three heuristic rules to enhance the learning algorithm of FMM are introduced. First, a new hyperbox expansion rule is introduced to eliminate the overlapping problem during the hyperbox expansion process. Second, the existing hyperbox overlap test rule is extended by discovering other possible overlapping cases. Third, a new hyperbox contraction rule to resolve possible overlapping cases is provided. The effectiveness of the algorithm is evaluated using benchmark data sets and a real medical diagnosis task. The results are better than various popular existing classifiers

Modified FMM neural network (MFMMN), novel classification model to perform the supervised classification of data is proposed in [26]. The FMM neural network can process only continuous attribute values and cannot handle the discrete values. Moreover the rationalization of the classification results given is required to be obtained to make it more applicable to the real world applications. These issues are attended and solved in the proposed MFMMN algorithm. In the MFMMN, each hyperbox have min-max values defined in terms of continuous attributes and a set of binary strings defined for discrete attributes. Bitwise 'and' and 'or' operators are used to adjust the discrete values associated with each hyperbox. The pruning removes the less useful hyperboxes based on their confidence factor. The experimentation with nine different datasets taken from the University of California, Irvine (UCI) machine learning repository and the case study of a real time weather data is evaluated. The proposed model provides very good accuracy. Further, the number of hyperboxes obtained after pruning are very less leading to less number of concise rules and hence the reduced computational complexity.

\section{FNNS FOR CLUSTERING}

The FMM clustering neural network also [3] uses hyperbox fuzzy sets and learning consists of creating, expanding and contracting hyperboxes in the pattern space. The learning begins by inputting an input pattern and finding the nearest hyperbox to that pattern that can expand (if necessary) to include the pattern. If a hyperbox cannot be found that meets the expansion criteria, a new hyperbox is created and added to the system. This augmentation process permits existing clusters to be refined over time, and allows addition of new clusters without the need of retraining. One of the unwanted effects of hyperbox expansion is overlapping hyperboxes. This overlapping causes ambiguity because patterns falling in the overlapping area fully belong to two or more different clusters. A contraction removes the overlap by employing the minimum disturbance principle.

The trained network consists of two layers; input layer, hyperbox nodes layer. The input layer nodes do not do any processing. These nodes only forward the input to their outputs. The hyperbox nodes represent clusters and use the fuzzy membership function to deliver its output. The output is soft decision indicating to which cluster the input pattern belongs. This soft decision can be converted to hard decision.

In [7] authors have proposed fuzzy hyperline segment clustering neural network (FHLSCNN). The proposed algorithm utilizes hyperline segment (HLS) fuzzy sets instead of fuzzy set hyperboxes. The algorithm consists of four steps: creation of HLSs, bunching of HLSs, overlap test and overlap removal. The experimentation with Fisher Iris data set shows that the proposed approach outperforms compared to original FMM clustering neural network.

Authors in [9] have proposed another FNN, fuzzy mean point clustering neural network successfully applied for clustering application. The fuzzy clustering and neural network [19] is employed effectively for uncertainty information interpretation of hyperspectral images.

\section{GENERAL FNNs}

The general FNNs are the algorithms which take care of labeled and unlabeled patterns that are applied during training. These FNNs incorporate fusion of supervised and unsupervised paradigms. Therefore the single algorithm can be used for pure classification, pure clustering or cross of classification and clustering.

The general fuzzy min-max (GFMM) neural network is a generalization and extension of the fuzzy min-max clustering and classification algorithms developed by Simpson [5]. The GFMM algorithm combines the supervised and unsupervised learning within a single training algorithm. This combination can be used as pure clustering, pure classification, or hybrid clustering classification. This fusion exhibits an interesting property of finding decision boundaries between classes and clusters during training. In GFMM algorithm also the hyperbox fuzzy sets are used for representation of clusters and classes.

Learning requires a few passes through the data. It also consists of placing and adjusting the hyperboxes in the pattern space which is referred to as an expansion-contraction process which is similar as with original algorithms proposed by Simpson. The classification results are fuzzy and if required can be converted to crisp decisions. Learning allows incorporation of new data without the need for retraining.

The GFMM algorithm preserves few remarkable features of the original algorithms. However, a number of modifications also have been proposed. These modifications include new definition of input in order to have room for fuzzy input patterns in the form of lower and upper bounds, fusion of the supervised and unsupervised learning, new well behaving membership function, modified hyperbox expansion criterion and incredible learning algorithm to tackle with labeled and unlabeled data patterns emerging during training.

A detailed explanation of the GFMM neural network, its evaluation with the original Simpson's fuzzy min-max neural networks is given. The paper also describes learning algorithm with 2-D example. In this experiment, two dimensional data set 
which is a mixture of an unlabeled and labeled samples is considered during training. The example describes learning algorithm clearly. The application to the leakage detection and identification in water distribution systems is also given.

The general fuzzy hyperline segment neural network (GFHLSNN) [10] is an extension of the fuzzy hyperline segment neural network in [8]. The fusion of supervised and unsupervised learning in a single algorithm can be used for pure classification, pure clustering and hybrid classification and clustering. The approach utilizes fuzzy hyperline segments. The hyperline segment is defined by its two end points. A new membership function is introduced for fuzzy hyperline segment. The algorithm consists of steps to create and expand hyperline segments during training, to detect intersection of hyperline segments and removal of intersection. Algorithm is suitably modified for working with mixed data. The probability of intersection of hyperline segments decreases with the increase in the dimension of pattern space. Therefore, in $n$ dimensional space where $n$ is sufficiently large the learning is possible without intersection test and its removal. Therefore, like FHLSNN, the GFHLSNN is also quick in learning and very efficient algorithm.

The contribution in [13] is general fuzzy hypersphere neural network (GFHSNN) that uses supervised and unsupervised learning within a single training algorithm. It is an extension (FHSNN) [6] and can be used for pure classification, pure clustering or hybrid clustering and classification. This approach utilizes fuzzy hypersphere sets replacing fuzzy set hyperboxes as in original FMM neural networks and its variants. The fuzzy hypersphere is characterized by its center, a radius and a membership function. The algorithm consists of steps to create and expand hyperspheres, to detect overlap between hyperboxes and removal of overlap with suitable modification required to take care of mixed data.

Table 1. Summary of FNNs for Classification

\begin{tabular}{|l|l|}
\hline $\begin{array}{l}\text { FNN Model for } \\
\text { Classification }\end{array}$ & Attributes of Model \\
\hline $\begin{array}{l}\text { Fuzzy min-max } \\
\text { neural network for } \\
\text { classification [2] }\end{array}$ & $\begin{array}{l}\text { employs supervised learning, uses } \\
\text { fuzzy set hyperboxes for } \\
\text { representation of classes, supports } \\
\text { online training, quick learning, } \\
\text { provides soft decision }\end{array}$ \\
\hline $\begin{array}{l}\text { Fuzzy neural } \\
\text { networks for } \\
\text { classification and } \\
\text { detection of } \\
\text { anomalies [4] }\end{array}$ & $\begin{array}{l}\text { performance, the classification results } \\
\text { are independent of the presentation } \\
\text { order }\end{array}$ \\
\hline $\begin{array}{l}\text { Fuzzy hypersphere } \\
\text { neural network } \\
\text { classifier [6] }\end{array}$ & $\begin{array}{l}\text { employs supervised learning, uses } \\
\text { fuzzy set hyperspheres for } \\
\text { representation of classes, supports } \\
\text { online training, quick learning, } \\
\text { provides soft decision }\end{array}$ \\
\hline $\begin{array}{l}\text { Fuzzy hyperline } \\
\text { segment neural } \\
\text { network [8] }\end{array}$ & $\begin{array}{l}\text { employs supervised learning, uses } \\
\text { fuzzy set hyperline segments for } \\
\text { representation of classes, supports } \\
\text { online training, quick learning, } \\
\text { exceptionally quick in recall, } \\
\text { provides soft decision }\end{array}$ \\
\hline
\end{tabular}

\begin{tabular}{|c|c|}
\hline $\begin{array}{l}\text { Modified fuzzy } \\
\text { hypersphere neural } \\
\text { network classifier } \\
{[11]}\end{array}$ & $\begin{array}{l}\text { employs supervised learning, uses } \\
\text { fuzzy set hyperspheres, modified } \\
\text { membership function, supports online } \\
\text { training, quick learning, provides soft } \\
\text { decision }\end{array}$ \\
\hline $\begin{array}{l}\text { Modified fuzzy } \\
\text { hyperline segment } \\
\text { neural network } \\
{[14]}\end{array}$ & $\begin{array}{l}\text { supervised learning, uses fuzzy set } \\
\text { hyperline segments, online training, } \\
\text { modified membership function, quick } \\
\text { learning, exceptionally quick in } \\
\text { recall, provides soft decision }\end{array}$ \\
\hline $\begin{array}{l}\text { Modular Fuzzy } \\
\text { Hypersphere } \\
\text { Neural Network } \\
{[15]}\end{array}$ & $\begin{array}{l}\text { offers higher degree of parallelism, } \\
\text { each module exposed to the patterns } \\
\text { of only one class, extremely quick in } \\
\text { training, suitable for big real } \\
\text { database, online training }\end{array}$ \\
\hline $\begin{array}{l}\text { A fuzzy min-max } \\
\text { neural network } \\
\text { classifier with } \\
\text { compensatory } \\
\text { neuron architecture } \\
{[18]}\end{array}$ & $\begin{array}{l}\text { uses hyperbox fuzzy sets, employs } \\
\text { new compensatory neuron } \\
\text { architecture, supports on line } \\
\text { adaptation in a single pass, yields } \\
\text { reduced classification and gradation } \\
\text { errors, performance is less dependent } \\
\text { on the initialization of maximum } \\
\text { hyperbox size coefficient }\end{array}$ \\
\hline $\begin{array}{l}\text { A modified fuzzy } \\
\text { min-max neural } \\
\text { network with rule } \\
\text { extraction [20] }\end{array}$ & $\begin{array}{l}\text { uses an Euclidean distance measure } \\
\text { for prediction, a rule extraction } \\
\text { algorithm, uses pruning }\end{array}$ \\
\hline $\begin{array}{l}\text { A modified fuzzy } \\
\text { min-max neural } \\
\text { network with A } \\
\text { genetic-algorithm- } \\
\text { based rule } \\
\text { extractor for } \\
\text { pattern } \\
\text { classification }[22]\end{array}$ & $\begin{array}{l}\text { a two-stage pattern classification and } \\
\text { rule extraction system, a modified } \\
\text { FMM neural network, utilizes } \\
\text { genetic-algorithm (GA)-based rule } \\
\text { extractor }\end{array}$ \\
\hline $\begin{array}{l}\text { Data-core-based } \\
\text { fuzzy min-max } \\
\text { neural network } \\
{[23]}\end{array}$ & $\begin{array}{l}\text { a new membership function, } \\
\text { contraction process is eliminated }\end{array}$ \\
\hline $\begin{array}{l}\text { A hybrid FMM- } \\
\text { CART model [24] }\end{array}$ & $\begin{array}{l}\text { utilizes FMM for classification and } \\
\text { CART for rule extraction, supports } \\
\text { offline and online properties }\end{array}$ \\
\hline $\begin{array}{l}\text { An enhanced fuzzy } \\
\text { min-max neural } \\
\text { Network for } \\
\text { pattern } \\
\text { classification [25] }\end{array}$ & $\begin{array}{l}\text { a new hyperbox expansion rule, } \\
\text { extended hyperbox overlap test rule, a } \\
\text { new hyperbox contraction rule }\end{array}$ \\
\hline $\begin{array}{l}\text { Modified fuzzy- } \\
\text { min max neural } \\
\text { network for data } \\
\text { with mixed } \\
\text { attributes [26] }\end{array}$ & $\begin{array}{l}\text { Allows discrete input, redefined } \\
\text { hyperbox representation, modified } \\
\text { membership function and learning, } \\
\text { utilizes pruning, to the real world } \\
\text { applications }\end{array}$ \\
\hline
\end{tabular}


Moreover, Modular general fuzzy hypersphere neural network in [27] the contribution in [13] is extended further proposing modular approach which leads in decrease of computational complexity due to parallelism. Tables I, II and III gives a summary of FNNs discussed in the sections II, III and IV, respectively.

Table 2. Summary of FNNs for Clustering

\begin{tabular}{|l|l|}
\hline $\begin{array}{l}\text { FNN Model for } \\
\text { Classification }\end{array}$ & Attributes of Model \\
\hline $\begin{array}{l}\text { Fuzzy min-max } \\
\text { neural network for } \\
\text { clustering [3] }\end{array}$ & $\begin{array}{l}\text { employs unsupervised learning, uses } \\
\text { fuzzy set hyperboxes for } \\
\text { representation of clusters, supports } \\
\text { online training, quick learning }\end{array}$ \\
\hline $\begin{array}{l}\text { Fuzzy hyperline } \\
\text { segment clustering } \\
\text { neural network [7] }\end{array}$ & $\begin{array}{l}\text { Utilizes unsupervised learning, uses } \\
\text { fuzzy set hyperline segments for } \\
\text { representation of classes, supports } \\
\text { online training, quick learning, } \\
\text { exceptionally quick in recall }\end{array}$ \\
\hline $\begin{array}{l}\text { Fuzzy Mean Point } \\
\text { Clustering Neural } \\
\text { Network [9] }\end{array}$ & $\begin{array}{l}\text { uses fuzzy sets, computation of mean } \\
\text { for centre of clusters }\end{array}$ \\
\hline $\begin{array}{l}\text { Fuzzy clustering } \\
\text { and neural network } \\
\text { [19] }\end{array}$ & $\begin{array}{l}\text { fuzzy clustering and neural network, } \\
\text { uncertainty information interpretation }\end{array}$ \\
\hline
\end{tabular}

Table 3. Summary of General FNNs

\begin{tabular}{|c|c|}
\hline $\begin{array}{l}\text { FNN Model for } \\
\text { Classification }\end{array}$ & Attributes of Model \\
\hline $\begin{array}{l}\text { General fuzzy min- } \\
\text { max neural } \\
\text { network for } \\
\text { clustering and } \\
\text { classification [5] }\end{array}$ & $\begin{array}{l}\text { generalization and extension of FMM } \\
\text { algorithms, modified hyperbox } \\
\text { membership function and input } \\
\text { representation, modified learning to } \\
\text { deal with labeled and unlabeled } \\
\text { samples, fusion of unsupervised and } \\
\text { supervised training, pure } \\
\text { classification, pure clustering, hybrid } \\
\text { classification and clustering }\end{array}$ \\
\hline $\begin{array}{l}\text { General Fuzzy } \\
\text { Hyperline Segment } \\
\text { Neural Network } \\
{[10]}\end{array}$ & $\begin{array}{l}\text { generalization and extension of } \\
\text { FHLSNN algorithm, utilizes fuzzy } \\
\text { hyperline segments, modified } \\
\text { learning to deal with labeled and } \\
\text { unlabeled samples, blending of } \\
\text { unsupervised and supervised training, } \\
\text { pure classification, pure clustering, } \\
\text { hybrid classification and clustering }\end{array}$ \\
\hline $\begin{array}{l}\text { General fuzzy } \\
\text { hypersphere neural } \\
\text { network [13] }\end{array}$ & $\begin{array}{l}\text { generalization and extension of } \\
\text { FHSNN algorithm, utilizes fuzzy } \\
\text { hyperspheres, modified learning to } \\
\text { deal with labeled and unlabeled } \\
\text { samples, blending of unsupervised } \\
\text { and supervised training, pure } \\
\text { classification, pure clustering, hybrid } \\
\text { classification and clustering }\end{array}$ \\
\hline $\begin{array}{l}\text { Modular general } \\
\text { fuzzy hypersphere } \\
\text { neural network } \\
{[19]}\end{array}$ & $\begin{array}{l}\text { modular approach offers higher } \\
\text { degree of parallelism with fusion of } \\
\text { supervised and unsupervised } \\
\text { paradigms, online training, very } \\
\text { learning, suitable for big real database }\end{array}$ \\
\hline
\end{tabular}

\section{CONCLUSIONS}

This paper provides review on recent FNNs focusing on pattern classification and clustering. There are a variety of traditional techniques to deal with pattern classification and clustering. However, the recent FNNs that have been proposed, provide a better choice for pattern classification and clustering.

The review is provided by grouping FNNs in three categories. Supervised FNNs can be used only for classification tasks. Likewise, unsupervised FNNs can be used for clustering. However, general FNNs with fusion of supervised and unsupervised training are suitable for pure classification, pure clustering and hybrid classification and clustering.

There are many contributions from various researchers that exploit basic approaches proposed by Simpson. The proposed alterations in various variants include modification of fuzzy hyperbox membership function, replacing hyperbox by hypersphere or hyperline segment and allowing labeled and unlabelled samples during training.

Distinct from other techniques, the FNNs are efficient, support online adaptation without need of retraining, provides fuzzy decision, suitable for linearly non-separable pattern classification and clustering, allows validation of performance, and provides good generalization and quick recall. Therefore, FNNs are extraordinarily suitable for difficult real world problems involving classification and clustering.

However, it is observed that all the authors claim that the learning allows incorporation of new data without the need for retraining ignoring the fact that the training may require few passes by adjusting the value of tuning parameter(s).

Therefore, there is scope for further research leading to design of FNN allowing single pass training through the data and suitable for online adaptation. In a true sense such a FNN is suitable for real time pattern classification and clustering applications.

\section{REFERENCES}

[1] Zurada, J. M. 1994. Introduction to Artificial Neural Systems, Bombay: Jaico Publishing House.

[2] Simpson, P. K. 1992. Fuzzy min-max neural networks Part 1: Classification. IEEE Trans. on Neural Networks. Vol. 3. No. 5. 776-786.

[3] Simpson, P. K. 1993. Fuzzy min-max neural networks Part 2: Clustering. IEEE Transactions on Fuzzy Systems. Vol. 1. No. 1. 32-45.

[4] Meneganti, M. Saviello, F. S. and Tagliaferri, R. 1998. Fuzzy neural networks for classification and detection of anomalies. IEEE Transactions on Neural Networks. Vol. 9. No. 5. 848-861.

[5] Gabrys, B. and Bargiela, A. 2000. General fuzzy min-max neural network for clustering and classification. IEEE Transactions on Neural Networks. Vol. 11. No. 3. 769783.

[6] Kulkarni, U. V. and Sontakke, T. R. 2001. Fuzzy hypersphere neural network classifier. IEEE International Fuzzy Systems Conference. Melbourne, Australia. 1559. 1562.

[7] Kulkarni, U. V. Sontakke, T. R. and Kulkarni, A. B. 2001. Fuzzy hyperline segment clustering neural network. Electronics Letters. Vol. 37. No. 5. 301-303. 
[8] Kulkarni, U. V. Sontakke, T. R. and Randale, G. D. 2001 Fuzzy hyperline segment neural network for rotation invariant handwritten character recognition. In Proc. Joint Int. Conference on Neural Networks, Washington DC, USA, (IEEE: INNS: IJCNN 2001), Vol. 4. 2918-2923.

[9] Patil, P.M. Kulkarni, U.V. and Sontakke, T.R. 2002. Fuzzy Mean Point Clustering Neural Network. Proceedings of the International Conference on Neural Information Processing. Vol. 2. 871-875.

[10] Patil, P.M. Kulkarni, U.V. and Sontakke, T.R. 2002 General Fuzzy Hyperline Segment Neural Network. Proceedings of the IEEE International Conference on Systems, Man and Cybernetics. Vol. 4.

[11] Doye, D.D. Kulkarni, U.V. and Sontakke, T.R. 2002. Speech recognition using modified fuzzy hypersphere neural network. Proceedings of the IEEE/INNS International Joint Conference on Neural Networks. Vol. 1. 12-17.

[12] Gabrys, B. 2002. Combining neuro-fuzzy classifiers for improved generalization and reliability. Proceedings of the IEEE/INNS International Joint Conference on Neural Networks. Vol. 3. 2410 - 2415.

[13] Kulkarni, U.V. Doye, D.D. and Sontakke, T.R. 2002. General fuzzy hypersphere neural network.Proceedings of the IEEE/INNS International Joint Conference on Neural Networks. Vol. 3. 2369-2374.

[14] Patil, P.M. Dhabe, P.S. Kulkarni, U.V. and Sontakke, T.R. 2003. Recognition of handwritten characters using modified fuzzy hyperline segment neural network. Proceedings of the IEEE International Conference on Fuzzy Systems. Vol. 2. 1418-1422. 2003.

[15] Patil, P.M. Kulkarni, U.V. and Sontakke, T.R. 2003. Modular Fuzzy Hypersphere Neural Network. Proceedings of the IEEE International Conference on Fuzzy Systems. Vol. 1. 232-236.

[16] Nandedkar, A. V. and Biswas, P. K. 2004. A Fuzzy MinMax Neural Network Classifier With Compensatory Neuron Architecture. Proceedings of the 17th International Conference on Pattern Recognition. Vol. 4. 553-556.

[17] Nandedkar, A. V. and Biswas, P. K. 2006. A Reflex fuzzy min max neural network for granular data classification. Proceedings of the 18th International Conference on Pattern Recognition. Vol. 2. 650-653.
[18] Nandedkar, A.V. and Biswas, P.K. 2007. A fuzzy min-max neural network classifier with compensatory neuron architecture. IEEE transactions on neural networks and learning systems. Vol. 18. No. 1.

[19] Li, H. L. Zhu, H. and Liu, G. 2008. Hyperspectral images for uncertainty information interpretation based on fuzzy clustering and neural network. The Int. Archives of the Photogrammetry, Remote Sensing and Spatial Information Sciences. Vol. 1. No. 37. 307-312.

[20] Anas Quteishat, Chee Peng Lim. 2008. A modified fuzzy min-max neural network with rule extraction and its application to fault detection and classification. Applied Soft Computing. Science Direct. Elsevier Publication.

[21] Chaudhari, B.M., Barhate, A.A. and Bhole, A.A. 2009 Signature recognition using fuzzy min-max neural network. Proceedings of the International Conference on Control. Automation, Communication and Energy Conservation. 1-7.

[22] Anas, Q. Chee, P.L. and Kay, S. T. 2010. A modified fuzzy min-max neural network with a genetic algorithm based rule extractor for pattern classification. IEEE transactions on Systems, Man, and Cybernetics. Vol. 40.

[23] Huaguang Zhang, Jinhai Liu, Dazhong Ma, and Zhanshan, W. 2011. Data-core-based fuzzy min-max neural network for pattern classification. IEEE transactions on neural networks. Vol. 22. No. 12

[24] Manjeevan Seera, Chee Peng Lim, Dahaman Ishak, and Harapajan Singh. 2012. Fault detection and diagnosis of induction motors using motor current signature analysis and a hybrid FMM-CART model. IEEE transactions on neural networks and learning systems. Vol. 23. No. 1.

[25] Mohammed and Chee Peng Lim. 2015. An enhanced fuzzy min-max neural Network for pattern classification. IEEE transactions on neural networks and learning systems. Vol. 26. No. 3 .

[26] Shinde, Swati and Kulkarni, Uday. 2016. Extracting classification rules from modified fuzzy-min max neural network for data with mixed attributes. Applied Soft Computing. Science Direct. Elsevier Publication. 40364 378

[27] Patil, P.M. Kulkarni, S.N. Kulkarni, U.V. and Sontakke, T.R. 2005. Modular general fuzzy hypersphere neural network. Proceedings of the 17th IEEE Int. Conf. on Tools with Artificial Intelligence (ICTAI'05). 1082-3409/05. 